\title{
A produção de soja como um vetor para a sustentabilidade: uma abordagem econométrica para o Mato Grosso
}

\author{
Soy production as a vector for sustainability: an econometric approach for Mato Grosso
}

La producción de soja como vector de sostenibilidad: un enfoque econométrico para Mato Grosso

Recebido: 04/10/2021 | Revisado: 10/10/2021 | Aceito: 11/10/2021 | Publicado: 12/10/2021

\author{
Ingrid Lorrane Miranda de Sousa \\ ORCID: https://orcid.org/0000-0001-5592-7477 \\ Universidade Federal do Oeste do Pará, Brasil \\ E-mail: estevgremio@gmail.com \\ Brena do Nascimento Carvalho \\ ORCID: https://orcid.org/0000-0002-0660-8514 \\ Universidade do Estado do Amazonas, Brasil \\ E-mail: brenanc16@gmail.com \\ Tarcísio da Costa Lobato \\ ORCID: https://orcid.org/0000-0002-2002-5622 \\ Universidade Federal do Oeste do Pará, Brasil \\ E-mail: tarcisio.lobato@ufopa.edu.br
}

\begin{abstract}
Resumo
Um dos obstáculos para alcançar o desenvolvimento sustentável é o de formular mecanismos de mensuração, tais como os indicadores de sustentabilidade, os quais se constituem como instrumentos fundamentais para acompanhar e avaliar o progresso alcançado em direção ao desenvolvimento sustentável. O objetivo deste artigo é analisar como a produção de soja influência na sustentabilidade dos municípios do Estado do Mato Grosso, por meio da construção de um Índice de Sustentabilidade da Produção da Soja ISPS no ano de 2010. Os resultados demonstraram que a dimensão social foi a que mais explicou a variabilidade total dos dados. Em relação aos impactos individuais dos indicadores, constatou-se que a área colhida é a que mais influência na variabilidade do ISPS, seguido do Plano de Recuperação de Áreas Degradadas e vulnerável a pobreza. Neste estudo, conclui-se por meio do Índice de Sustentabilidade da Produção da Soja proposto, que a produção de soja contribui para a sustentabilidade dos municípios do Estado do Mato Grosso, uma vez que os municípios com maiores produções de soja como Sorriso, Lucas do Rio Verde, Campo Novo do Parecis apresentam um nível alto de sustentabilidade.
\end{abstract}

Palavras-chave: Índice de sustentabilidade; Produção de soja; Análise fatorial; Mato Grosso.

\begin{abstract}
One of the obstacles to achieving sustainable development is to formulate measurement mechanisms, such as sustainability indicators, which are key tools for monitoring and assessing progress towards sustainable development. The aim of this article is to analyze how soybean production influences the sustainability of municipalities in the State of Mato Grosso, through the construction of an ISPS Soybean Production Sustainability Index in 2010. The results showed that the social dimension was the one that most explained the total variability of the data. In relation to the individual impacts of the indicators, it was found that the harvested area is the one that most influences the variability of ISPS, followed by the Recovery Plan for Degraded areas and vulnerable to poverty. In this study, it is concluded through the proposed Soybean Production Sustainability Index, that soybean production contributes to the sustainability of the municipalities of the State of Mato Grosso, once the municipalities with higher soybean production such as Sorriso, Lucas do Rio Verde, Campo Novo do Parecis have a high level of sustainability.
\end{abstract}

Keywords: Sustainability index; Soybean production; Factor analysis; Mato Grosso.

\section{Resumen}

Uno de los obstáculos para lograr el desarrollo sostenible es formular mecanismos de medición, como los indicadores de sostenibilidad, que son herramientas clave para monitorear y evaluar el progreso hacia el desarrollo sostenible. El objetivo de este artículo es analizar cómo la producción de soja influye en la sostenibilidad de los municipios del Estado de Mato Grosso, a través de la construcción de un Índice de Sostenibilidad de la Producción de Soja ISPS en 2010. Los resultados mostraron que la dimensión social fue la que más explicó la variabilidad total de los datos. En relación a los impactos individuales de los indicadores, se encontró que el área cosechada es la que más influye en la variabilidad de los ISPS, seguido del Plan de Recuperación para zonas degradadas y vulnerables a la pobreza. En este estudio, se concluye a través del propuesto Índice de Sostenibilidad de la Producción de Soja, que la producción de soja contribuye a la sostenibilidad de los municipios de la Estado de Mato Grosso, ya que los municipios con mayores 
sumas de producción de soja como Sorriso, Lucas do Rio Verde, Campo Novo do Parecis tienen un alto nivel de sostenibilidad.

Palabras clave: Índice de sostenibilidad; Producción de soja; Análisis factorial; Mato Grosso.

\section{Introdução}

O agronegócio é, atualmente, um setor fundamental para a economia brasileira, isso se deve especialmente a sua capacidade de sustentar a economia em períodos difíceis e contribuir para a retomada do crescimento econômico do país. É importante frisar que o Brasil é o terceiro maior exportador mundial de produtos do agronegócio devido possuir recursos naturais abundantes, ao aumento da produtividade, câmbio favorável, grande demanda dos países asiáticos por produtos agropecuários, tal como a China, inovações tecnológicas e as linhas de financiamento do governo federal (ASSAD et al., 2012). Contudo, associado ao crescimento econômico aumentam as pressões para que o setor minimize os seus impactos no meio ambiente e que seja um indutor do desenvolvimento socioeconômico nas regiões em que é realizado (Hirakuri et. al, 2014).

Nesse cenário, o agronegócio, composto por insumos para a agropecuária, produção agropecuária primária, agroindústria e agrosserviços, representa aproximadamente 23\% do PIB nacional (CNA, 2017). Conforme o Ministério da Agricultura, Pecuária e Abastecimento - MAPA (2017), as exportações brasileiras do agronegócio alcançaram US\$ 56,395 bilhões e as importações atingiram US\$ 8,348 bilhões, gerando um superávit na balança comercial do agronegócio de US\$ 48,047 bilhões (janeiro/2017 a julho/2017). O complexo soja liderou as exportações totais com US\$23,012 bilhões, representando, nesse período, 40,80\% das exportações do agronegócio, sendo, portanto, uma cadeia produtiva essencial para o superávit na balança comercial brasileira.

Neste cenário, torna-se essencial medir os impactos que a produção da soja possa acarretar para o meio ambiente, alertando para medidas que visem a sustentabilidade em sua produção. A importância da sustentabilidade será mais bem compreendida quando for possível proceder à sua avaliação entre os diferentes setores ou sistemas, com o desígnio de identificar qual o mais sustentável, de modo a permitir que se encontrem soluções para os problemas observados. Num mundo onde a agricultura se tornou globalizada, faz-se necessário mensurar a sustentabilidade das atividades, cadeias produtivas, regiões e países (Costa, 2010).

A sustentabilidade pode ser mensurada a partir de indicadores que contemplem, sobretudo, metodologia, relevância e viabilidade e que a sua seleção compreenda clareza na delimitação dos objetivos da pesquisa, os custos reais no desenvolvimento, elevada qualidade e confiabilidade (IBGE, 2015). Assim, iniciativas que busquem a produção agrícola de forma sustentável tem sido o foco de vários estudos acadêmicos.

A análise de cadeias produtivas, como a da soja, a partir dos indicadores de sustentabilidade auxilia na orientação de pesquisas, políticas públicas, transferência de tecnologia e na assistência técnica, a fim de sanar possíveis entraves de sustentabilidade. Deste modo, um Índice Integrado de Sustentabilidade, composto por indicadores das esferas ambientais, econômicos e sociais, viabiliza identificar o nível da sustentabilidade da cadeia produtiva na região avaliada. Dessa forma, ao utilizar esse índice se obtém coeficientes dimensionais que permitem verificar se a produção de soja possui um alto, médio, baixo ou um crítico nível de sustentabilidade, evidenciando as suas principais fragilidades e potencialidades (Hirakuri et. al., 2015).

Diante disso, surge a necessidade de elaborar uma metodologia confiável, relevante e viável para mensurar a sustentabilidade da produção de soja, tendo em vista que está é considerada por vários autores como um entrave para a sustentabilidade do país, assim, o desenvolvimento desta pesquisa se desdobra num questionamento central, qual seja, saber se a produção da soja se estabelece de forma sustentável no estado do Mato Grosso. 


\section{Fundamentação Teórica}

\subsection{Desenvolvimento sustentável e a produção de soja no Mato Grosso}

Conforme Jacobi (1999), a problemática da sustentabilidade adquiriu um papel central na reflexão acerca das dimensões do desenvolvimento e das alternativas que se configuram na segunda metade do século XX quando o cenário socioambiental demonstrava que os impactos sobre o meio ambiente estavam se transformando mais complexos. Diante disso, conferências e acordos vêm sendo realizados com a finalidade de debater e apresentar alternativas de conciliar o crescimento econômico e a conservação ambiental a partir de um desenvolvimento sustentável.

A definição de desenvolvimento sustentável foi estabelecida a priori pela Comissão Mundial sobre o Meio Ambiente e Desenvolvimento, na Assembléia Geral das Nações Unidas, em 1987. Esta Comissão produziu o Relatório Brundtland ou Relatório Nosso Futuro Comum, como é usualmente conhecido, onde o desenvolvimento sustentável é apresentado como "o desenvolvimento capaz de suprir as necessidades da geração atual, sem comprometer a capacidade de atender as necessidades das futuras gerações" (Mueller, 1995, p.66).

O desenvolvimento sustentável abrange três macro temas, tais como os aspectos ambientais, sociais e econômicos. Dessa forma, refere-se a estratégias que promovam a viabilidade ambiental e socioeconômica, refutando, assim, a percepção de que tal desenvolvimento está estritamente associado apenas às alterações ecológicas resultantes dos processos sociais. Devido dispor de um conceito muito amplo, o desenvolvimento sustentável pode ser utilizado pelos diferentes âmbitos da sociedade, como pelo setor governamental nos níveis nacional, regional e local, e pelo setor produtivo, uma vez que a sinergia entre esses aspectos permite a aplicação do conceito de Desenvolvimento Sustentável, ou Sustentabilidade, onde quer que ele seja aplicado (Jacobi, 1999).

Diante deste cenário, o agronegócio por se utilizar do desmatamento, degradação dos solos e maior dose de fertilizantes e agrotóxicos, impõe diversos efeitos questionáveis a respeito de uma produção que preze pela sustentabilidade. Portanto, como uma tentativa de minimizar os impactos negativos dessa modernização da agricultura ocorrido na Amazônia, onde a produção de soja estava se intensificando e contribuindo significativamente para o aumento exacerbado do desmatamento, foi criada a Moratória da soja em julho de 2006, devido a pressões de ambientalistas e do comércio exterior para travar o desmatamento com a finalidade da produção de soja na Amazônia brasileira (Kastens et. al., 2017).

A moratória afetou a relação entre os importadores e o setor do agronegócio brasileiro, que sentiu necessidade de demonstrar que seus produtos são produzidos de forma sustentável. Assim, a Associação Brasileira das Indústrias de Óleos Vegetais Indústrias (ABIOVE) e a Associação de Exportadores de Cereais no Brasil (ANEC), contrataram uma empresa para monitorar o desmatamento de áreas maiores que 100ha em municípios com mais de 5000 ha de soja plantada no bioma amazônico nos estados de Mato Grosso, Pará, Rondônia, Roraima e Amapá, inicialmente, a área monitorada incluiu 37 municípios (ABIOVE, 2017).

Nesse contexto, ao analisar a relação entre a sustentabilidade e o setor do agronegócio, verifica-se que este setor é considerado um grande vilão das problemáticas ambientais e sociais, tendo em vista que a sua evolução decorreu da expansão das fronteiras agrícolas, do êxodo rural e da má utilização dos recursos naturais. Além disso, introduziu uma nova perspectiva de como produzir, pois o campo abriu espaço para uma agricultura mecanizada, num movimento orquestrado que repassa ao urbano o problema de suas externalidades, tornando-se, portanto, um exemplo de mau desenvolvimento (Mueller, 1995).

Entretanto, a sua magnitude tem contribuído para ancorar a economia brasileira inclusive em períodos de recessão. Atualmente, em razão dos setores industrial, construção e serviços estarem fragilizados com a desaceleração da economia, o agronegócio se destaca como o único setor que apresenta condições de estimular o crescimento econômico, exceto quando ocorrem eventos climáticos extremos, tal como o verificado em 2016 que causou prejuízos para o setor (Barros, 2016). 
No agronegócio, dentre as commodities mais importante desta atividade a produção de soja, em virtude da produção nacional e exportações, sendo a maior produção efetuada pelo Mato Grosso. De acordo o MAPA (2019), o Mato Grosso liderou a produção na safra 2018/19, detém a produção de 32.134 mil toneladas, referente a 28,1\% do total produzido no país. Dessa forma, a economia do Mato Grosso se baseia predominantemente pela dinâmica do agronegócio, que opera com padrões internacionais de qualidade, em razão dos elevados índices de produtividade associados à fatores climáticos e tecnologias.

Com o escopo de fomentar a sustentabilidade na produção de soja do Estado, a Associação dos Produtores de Soja e Milho de Mato Grosso - APROSOJA com a parceria de órgãos como a Associação Brasileira da Indústria de Óleos Vegetais ABIOVE, o Serviço Nacional de Aprendizagem Rural - Senar/MT, entre outros, lançou em 2011, o Soja Plus, o qual é um programa de melhoria contínua orientado para o agricultor, que oferece aos agricultores de soja assistência na implementação da legislação ambiental e social brasileira, além de melhorar as práticas agrícolas, contribuindo para enfrentar os desafios de sustentabilidade identificados (Costa et al., 2015).

De acordo com Costa et al. (2015), esse programa é considerado uma ferramenta importante que apoia a implementação completa do Código Florestal e seus dois instrumentos fundamentais, o Cadastro Ambiental Rual - CAR e o Programa de Regularização Ambiental - PRA. Atualmente, com parcerias, inclusive, internacionais como a Iniciativa de Comércio Sustentável (IDH), o Soja Plus estabelece uma relação de trabalho direta e formal entre os produtores brasileiros de soja, os processadores e os principais compradores europeus de soja, compartilhando uma visão comum e um plano de ação para promover a produção responsável de soja no Brasil e usar na Europa.

\subsection{Dimensões dos indicadores de sustentabilidade}

Sequinel (2002) afirma que a noção de dimensões de sustentabilidade surgiu das bases conceituais da teoria de Sachs, o ecodesenvolvimento, a qual propunha a existência de cinco dimensões, tais como a ecológica, a social, a econômica, a cultural e a espacial. Ressalta-se que essas dimensões buscavam evidenciar a necessidade de tornar compatíveis a melhoria nos níveis de qualidade de vida e a preservação ambiental.

A partir dessa teoria, diversos autores passaram a estudar a sustentabilidade por intermédio da mensuração de indicadores, contudo, adotaram como pilares, especialmente, três dimensões, a econômica, a ambiental e a social, embora dentro dessas dimensões existam alguns indicadores que contemplariam outras dimensões, como a institucional, justificando, assim, a sua não inclusão (Sequinel, 2002).

Dessa forma, ao avaliar a sustentabilidade é essencial que as dimensões não sejam analisadas de forma dissociada uma da outra, tendo em vista que ao valorizar uma única dimensão as demais teriam os seus resultados negligenciados, e, partindo do pressuposto que muitas análises propostas possuem o intuito de norteara organização e dirigir planos políticos, uma análise fracionada e parcial produzirá resultados que podem impulsionar ações desacertadas (Orsi, 2009).

Nesse contexto, a dimensão ambiental é a mais amplamente defendida e discutida pela maioria dos autores, sendo difundida a partir das conferências e acordos realizados na década de 1970. Referindo-se a utilização dos recursos naturais e a degradação ambiental, está relacionada aos objetivos de preservação e conservação do meio ambiente, considerados fundamentais ao benefício das gerações futuras. Desta forma, seus defensores julgaram a necessidade de valorizar e controlar o uso dos recursos naturais, respeitando sua capacidade de recuperação em seu ciclo de vida (Quinhoneiro, 2015).

Neste aspecto, Quinhoeiro (2015) salienta que existe a necessidade de mudança na forma em que se analisa o fluxo de bens e serviços em dado sistema capitalista, considerando como parte de sua composição também os benefícios dos ativos ambientais. Fazendo com que a sociedade possua como objetivo a busca por um desenvolvimento adequado na qualidade do meio ambiente e da preservação dos recursos energéticos e naturais. Dessa forma, os indicadores da dimensão ambiental 
refletem os objetivos inerentes a preservação e conservação do meio ambiente, que segundo Martins \& Cândido (2008) são considerados essenciais para que haja a conservação da qualidade de vida e do ambiente para a atual e futuras gerações.

A dimensão econômica possui como objetivos a eficiência dos processos produtivos a partir das alterações nas estruturas de consumo orientadas a uma reprodução econômica sustentável a longo prazo. Essa dimensão deve levar em consideração que existem informações importantes a serem consideradas, não exclusivamente da manutenção do capital e as transações econômicas. Neste sentido, a economia deve proporcionar uma aplicação e um gerenciamento mais eficiente dos recursos e um fluxo regular de investimentos públicos e privados (Costa, 2010).

A dimensão social, por sua vez, representa, especialmente, os objetivos ligados ao consentimento das necessidades humanas, evolução da qualidade de vida e justiça social. Sachs (1993) sugere que se defina um processo de desenvolvimento que proporcione um crescimento estável com igualdade de renda, estimulando desta forma as diferenças sociais e a melhoria na qualidade de vida geral. Destaca-se que a base da sustentabilidade social, segundo Sachs, é alicerçada na diminuição das desigualdades.

\subsection{Indicadores de Desenvolvimento Sustentável do Mato Grosso}

Os Indicadores de Desenvolvimento Sustentável do Estado de Mato Grosso têm como bases metodológicas a Comissão para o Desenvolvimento Sustentável das Nações Unidas e a Comissão de desenvolvimento Sustentável da Comunidade Europeia. Além disso, utiliza o modelo Pressão - Estado - Resposta (PER), desenvolvido pela Organização para a Cooperação e Desenvolvimento Econômico - OCDE ao verificar como as atividades humanas impactam o ambiente e como a sociedade responde a esses impactos através de políticas ambientais, econômicas e setoriais (SEMA-MT, 2014).

Os indicadores de sustentabilidade foram criados por meio de uma matriz integrada de impactos, supondo para o meio natural os componentes: solo, relevo, regime hídrico, água, clima, ar, flora, fauna, paisagem, ecossistemas e substrato rochoso; à medida que para o meio humano foram utilizadas as variáveis: espaço urbano e periurbano, espaço de lazer e turismo, espaço florestal, espaço agrícola, patrimônio cultural, infraestrutura, economia, mercado de bens e serviços, e finanças públicas (SEMA-MT, 2014).

Como parte do processo de construção da matriz de identificação de impactos, fez-se necessário identificar as principais atividades desenvolvidas no Estado de Mato Grosso que gerariam impactos ambientais positivos ou negativos nos meios físico, biótico e antrópico. Para cada um dos impactos gerados pelas atividades foi estabelecido um peso que variou de 3 (impactos negativos) a +3 (impactos positivos) dependendo da sua abrangência e/ou sua intensidade (SEMA-MT, 2014).

Para a descrição dos impactos foram usados dados bibliográficos, bem como os gerados pela própria Secretaria de Estado e Meio Ambiente do Mato Grosso - SEMA MT pela implementação dos instrumentos de gestão ambiental, particularmente o licenciamento e o monitoramento. Na sequência, foi elaborada uma planilha contendo as dimensões, temas e subtemas tendo como base os utilizados pela Comissão de Desenvolvimento Sustentável da ONU e pelo IBGE. Desta forma, construiu-se uma relação entre os impactos levantados e as dimensões, temas e subtemas e identificados pela Comissão Técnica, os indicadores a serem monitorados que subsidiariam o acompanhamento e avaliação dos impactos gerados pelas atividades selecionadas (SEMA-MT, 2014).

Em suma, os Indicadores de Desenvolvimento Sustentável do MT são compostos por quatro dimensões, sendo que a dimensão ambiental contém 42 indicadores, a dimensão social compreende 49, a dimensão econômica abrange 23 , e a dimensão institucional apresenta 25, alcançando 139 indicadores (SEMA-MT, 2014).

Após a checagem da viabilidade dos indicadores, foi identificada a indisponibilidades de alguns deles, em função disso, a comissão teve que realizar um ajuste na relação dos indicadores. Para cada um dos indicadores foram produzidas planilhas contendo os dados por município do estado de Mato Grosso, preferencialmente para o ano de 2010; na 
impossibilidade de se conseguir os dados do referido ano, foram levantados dados de anos anteriores ou posteriores (SEMAMT, 2014).

\subsection{Metodologia para Avaliação de Sustentabilidade da Cadeia Produtiva da Soja no Brasil da Embrapa}

A metodologia elaborada pela Empresa Brasileira de Pesquisa Agropecuária - EMBRAPA em 2015 foi formulada a partir de dados secundários e com a contribuição de diversos pesquisadores de áreas correlatas da sustentabilidade como escopo de avaliar a sustentabilidade da cadeia produtiva da soja no Brasil através dos indicadores de sustentabilidade nas dimensões ambiental-agronômica, econômica e social.

Nesse contexto, essa metodologia possui 15 indicadores na dimensão ambiental-agronômica, 11 indicadores na dimensão econômica e sete indicadores na dimensão social, sendo que tais indicadores foram agrupados em diferentes atributos norteadores compondo um Índice Integrado de Sustentabilidade (Hirakuri et. al, 2015)

Os indicadores apresentam pesos distintos que variam de acordo com a sua importância e formam os três coeficientes dimensionais. Esses pesos foram estabelecidos por meio da Técnica Delphi, a qual consiste em um consenso entre especialistas no que tange a questões técnicas e cenários futuros, desse modo, especialistas de várias áreas de pesquisa com soja responderam dois questionários interativos para a definição da ordem e dos pesos dos indicadores (Hirakuri et.al, 2015).

A composição do Índice Integrado de Sustentabilidade fundamenta a discussão sobre os aspectos conjunturais essenciais para a sustentabilidade da cadeia produtiva da soja. Nessa perspectiva, através dos indicadores econômicos é possível verificar a viabilidade econômica da cadeia produtiva da soja, uma vez que estes utilizam atributos norteadores como a produção, produtividade, escoamento e exportação da soja.

Além disso, por meio dos indicadores sociais, especialmente averiguados com base no Índice de Desenvolvimento Humano - IDH e no Índice de Desenvolvimento Municipal - IFDM, investiga se a soja proporciona melhores condições de vida para população local quanto a educação, renda e saúde, e, os indicadores ambientais que permitem analisar a capacidade do ecossistema de se recompor mesmo com as interferências humanas.

Dessa forma, ao utilizar esses indicadores de sustentabilidade se obtém coeficientes dimensionais que permitem verificar se a cadeia produtiva da soja possui um alto, médio, baixo ou um crítico nível de sustentabilidade ambiental, econômica e social, como mostra a Tabela 1, evidenciando as suas principais fragilidades e potencialidades.

Tabela 1. Classificação da representação dos índices da EMBRAPA.

\begin{tabular}{ll}
\hline Índice & Performance \\
\hline IIS $>=80$ & Alto \\
$60<=$ IIS $<80$ & Médio \\
$40<=$ IIS $<60$ & Baixo \\
IIS $<=40$ & Nível crítico \\
\hline
\end{tabular}

Fonte: Autores.

\section{Procedimentos Metodológicos}

\subsection{Base de dados}

Para a construção do Índice de Sustentabilidade da Produção da Soja dos municípios Mato Grosso para o ano de 2010 , optou-se pela coleta dos dados secundários anuais obtidos nos sistemas de banco de dados do Instituto Brasileiro de Geografia e Estatística - IBGE, da Secretaria de Comércio Exterior - SECEX, do Atlas do Desenvolvimento Humano no Brasil, da 
Federação das Indústrias do Estado do Rio de Janeiro - FIRJAN, da Secretaria de Estado de Meio Ambiente do Mato Grosso SEMA-MT e da Secretaria de Estado de Planejamento do Mato Grosso - SEPLAN-MT.

Ressalta-se que esses indicadores foram selecionados com base nas metodologias dos Indicadores de Desenvolvimento Sustentável do IBGE, do Índice de Desenvolvimento Sustentável para Municípios de Martins e Cândido (2008), do Sistema de Indicadores de Desenvolvimento Sustentável do Governo do Estado do Mato Grosso e da Metodologia para Avaliação de Sustentabilidade da Cadeia Produtiva da Soja no Brasil da EMBRAPA.

Entretanto, ocorreu o problema relatado pelo IBGE (2015) com relação a coleta dos dados, existindo a presença de informações incompletas para os municípios, sendo que para se realizar o estudo com mais indicadores acarretaria na exclusão de mais da metade dos municípios no estudo, para contornar esse problema foram selecionados os indicadores que possuíam informações para o maior número de municípios, portanto, foi possível alcançar a marca de 92 municípios, o que representa uma amostra de cerca de 65\% da população, com os seguintes indicadores: Desmatamento, Área do município com Plano de Recuperação de Áreas Degradadas - PRAD, Área Plantada, Área Colhida, Quantidade Produzida, Rendimento Médio Da Produção, Valor Da Produção, Renda Per Capita, \% dos ocupados setor agropecuário com 18 anos ou mais, PIB preços correntes, Extremamente Pobre, Pobre, Vulnerável A Pobreza, Índice De Gini, IDH-M, IDH-M renda, IDH-M longevidade, IDH-M educação consolidaram a base de dados desse estudo.

\subsection{Instrumental analítico}

\subsubsection{Análise Fatorial (AF)}

A AF será utilizada para a construção do Índice Integrado de Sustentabilidade para Soja - IISS, essa técnica possibilita que se encontre uma estrutura em um conjunto de variáveis, assim como também é um processo de redução de dados. Esta técnica reduz p variáveis observáveis (Y1, Y2, Y3,..., Yp)T, retiradas de uma população, para número menor m de variáveis não observáveis independentes denominados fatores (F1, F2, F3,..., Fm) (Johnson; Wichern, 2007; Fávero et al. 2009, Ferreira, 2011).

O método de extração dos fatores foi por componentes principais e se baseia na decomposição espectral da matriz $\square$ a partir da matriz de autovalores e autovetores para a determinação das cargas fatoriais. Neste método, os componentes são calculados usando todas as variâncias das variáveis manifestas. (Johnson; Wichern, 2007).

O método de rotação ortogonal Varimax busca minimizar o número de variáveis que tem altas cargas em um fator, assim para cada fator existem apenas alguns pesos significativos e todos os outros sejam próximos de zero, facilitando a interpretação (Hair et al., 2005).

Para a verificação da adequação da análise foram utilizadas as Medida de Kaiser-Meyer-Olkin e teste de esfericidade de Bartlett, comunalidades e matriz de anti-imagem acima de 0,50 (Hair et al., 2005; Fávero et al., 2009).

\subsubsection{Construção do Índice de Sustentabilidade da Produção da Soja (ISPS)}

Nessa aplicação, espera-se que os fatores extraídos pela AF possam expressar as dimensões Social, Econômica e Ambiental, uma vez que se trata de uma variável latente, isto é, resultado de uma combinação linear dos indicadores de cada dimensão. Será possível analisar quais dessas dimensões mais estão contribuindo para a sustentabilidade, visto que o fator (dimensão) que explicar maior parte da variabilidade será o maior responsável pelos valores do índice.

Nesse contexto, pode-se selecionar dentre todos os indicadores apenas os que explicam a maior parte da variabilidade em conjunto entre eles, possibilitando eliminar os indicadores que não serão significativos para a construção do índice. Uma interpretação possível nessa etapa é verificar dentro de cada dimensão como os indicadores se relacionam entre si e como irão contribuir para o índice, podendo ser de forma positiva ou negativa. 
Diante dos resultados obtidos pela AF, o Índice de Sustentabilidade da Produção da Soja (ISPS) é definido como uma combinação dos escores fatoriais e a proporção da variância explicada por cada fator em relação à variância explicada por cada fator em relação à variância comum. Dessa forma, o IS adaptado de Carvalho et al. (2007), é dado por:

$$
I S=\sum_{j=1}^{q}\left(\frac{\lambda_{j}}{\Sigma_{j} \lambda_{j}} F P_{i j}\right), i=1,2, \ldots, n ;
$$

Em que $\lambda_{\mathrm{j}}$ é a variância explicada por causa do fator, $\Sigma_{j} \lambda$ é a soma total de variância explicada pelo conjunto de fatores comuns e $F P_{\mathrm{ij}}$ é o escore fatorial padronizado.

O escore fatorial é padronizado para se obter valores positivos dos escores originais e permitir a classificação dos solos, uma vez que os valores do ISPS estão situados entre zero e um.

$$
z=\frac{(x-\text { mínimo })}{\text { (máximo-minimo })}
$$

Para a interpretação dos resultados, será realizada a análise de cluster com o intuito de estabelecer quantos clusters serão construídos, desta forma será possível separar os municípios por grau de similaridade, portanto cada cluster terá um intervalo para o índice. Espera-se encontrar quatro clusters para que o índice de sustentabilidade classifique os municípios em situação Crítica, Alerta, Aceitável e Ideal.

\section{Resultados}

\subsection{Aplicação da Análise Fatorial}

Inicialmente foram utilizados os 18 indicadores mencionados na metodologia para aplicação da AF, com o intuito de se encontrar o melhor modelo para a construção do ISPS. Com a utilização de todas estas, após algumas tentativas para se encontrar a AF mais adequada, utilizando a matriz de anti-imagem e as comunalidades para expurgar os indicadores menos representativas, encontra-se necessário a retirada dos indicadores: rendimento médio da produção, \%dos ocupados do setore agropecuário com 18 anos ou mais, PIB preços correntes, índice de Gini e IDH-M longevidade. A tabela 2 apresenta os valores das comunalidades para os indicadores após aplicação da AF.

Vale destacar que os indicadores Desmatamento e Quantidade produzida apesar de apresentarem valores de comunalidades um pouco inferiores a 0,50 (Ver Tabela 2), optaram-se pela sua permanência ao considerar seus valores acima de 0,60 na matriz de anti-imagem e por sua importância teórica na composição do ISPS. Os resultados da aplicação da AF resultaram em um valor de 0,76 para o Teste de KMO e significativo para o teste de esfericidade de Bartlett. 
Tabela 2. Comunalidades para os indicadores.

\begin{tabular}{ll}
\hline Indicadores & Comunalidades \\
\hline PRAD & 0.83 \\
AP & 0.97 \\
AC & 0.97 \\
QP & 0.49 \\
VPR & 0.94 \\
EP & 0.70 \\
P & 0.88 \\
VP & 0.91 \\
IDHM & 0.91 \\
IDHM R & 0.85 \\
IDHM E & 0.69 \\
RPC & 0.82 \\
D & 0.39 \\
\hline
\end{tabular}

Fonte: Elaborado pelos autores.

Na Tabela 3 contém o resultado da solução inicial e rotacionada para os 13 fatores possíveis e seu relativo poder explanatório expresso pelos autovalores. Aplicando o critério do percentual de variância, três fatores foram extraídos e explicam cerca de $79,56 \%$ da variância total dos dados, contendo $46,53 \%$ da variabilidade no primeiro fator, $23,92 \%$ no segundo fator e $9,11 \%$ no terceiro fator.

Tabela 3. Análise da variação total explicada com a extração dos fatores.

\begin{tabular}{|c|c|c|c|c|c|c|}
\hline \multirow{3}{*}{ Componentes } & \multicolumn{3}{|c|}{ Autovalores e Variâncias Iniciais } & \multicolumn{3}{|c|}{ Variâncias após Rotação } \\
\hline & \multicolumn{3}{|l|}{ Variância } & \multicolumn{3}{|c|}{ Variância } \\
\hline & Total & $\%$ & $\%$ Acumulada & Total & $\%$ & $\%$ Acumulada \\
\hline 1 & 6,116 & 47,049 & 47,049 & 6,049 & 46,529 & 46,529 \\
\hline 2 & 3,164 & 24,342 & 71,391 & 3,109 & 23,916 & 70,445 \\
\hline 3 & 1,062 & 8,167 & 79,558 & 1,185 & 9,113 & 79,558 \\
\hline 4 & 0,795 & 6,116 & 85,674 & & & \\
\hline 5 & 0,793 & 6,100 & 91,774 & & & \\
\hline 6 & 0,453 & 3,485 & 95,259 & & & \\
\hline 7 & 0,395 & 3,040 & 98,298 & & & \\
\hline 8 & 0,105 & 0,807 & 99,105 & & & \\
\hline 9 & 0,072 & 0,551 & 99,657 & & & \\
\hline 10 & 0,021 & 0,160 & 99,817 & & & \\
\hline 11 & 0,016 & 0,123 & 99,939 & & & \\
\hline 12 & 0,007 & 0,057 & 99,996 & & & \\
\hline 13 & 0,000 & 0,004 & 100,000 & & & \\
\hline
\end{tabular}


Os fatores foram rotacionados utilizando o Varimax, segundo a Tabela 4 se pode alocar a qual fator cada variável pertence, analisando o maior valor em módulo dos escores dos componentes.

Tabela 4. Rotação dos fatores utilizando o método Varimax.

\begin{tabular}{llll}
\hline Indicadores & $\mathbf{1}$ & $\mathbf{2}$ & $\mathbf{3}$ \\
\hline PRAD & -0.07 & -0.14 & $\mathbf{- 0 . 9 0}$ \\
AP & -0.03 & $\mathbf{0 . 9 8}$ & 0.10 \\
AC & -0.03 & $\mathbf{0 . 9 8}$ & 0.10 \\
QP & $\mathbf{0 . 5 4}$ & 0.45 & -0.01 \\
VPR & -0.01 & $\mathbf{0 . 9 7}$ & 0.04 \\
EP & $\mathbf{- 0 . 8 3}$ & 0.06 & 0.10 \\
P & $\mathbf{- 0 . 9 3}$ & 0.01 & 0.14 \\
VP & $\mathbf{- 0 . 9 4}$ & -0.10 & 0.13 \\
IDHM & $\mathbf{0 . 9 5}$ & -0.04 & 0.02 \\
IDHM R & $\mathbf{0 . 9 1}$ & 0.05 & -0.16 \\
IDHM E & $\mathbf{0 . 8 0}$ & -0.12 & 0.18 \\
RPC & $\mathbf{0 . 9 0}$ & 0.07 & -0.11 \\
D & -0.39 & 0.05 & $\mathbf{0 . 4 9}$ \\
\hline
\end{tabular}

Fonte: Elaborado pelos autores.

O primeiro contém os indicadores QP, EP, P, VP, IDHM R, IDHM E e RP, representando a Dimensão Social. Apesar de a QP ser um indicador econômico, este apresenta grande importância teórica para a sustentabilidade, assim como se mostrou ao aparecer no fator principal. Nesse caso, essa dimensão é a que mais explica a variabilidade total dos dados, portanto esses indicadores quando analisados de forma conjunta exercem maior influência no ISPS do que os demais. Pode-se perceber que os indicadores com sinais negativos - Indicadores de pobreza - estão relacionados inversamente com os indicadores de desenvolvimento humano e renda per capita, ou seja, a medida que esses indicadores aumentam a pobreza tende a diminuir, assim como é constatado por Weinhold, Killick e Reis (2013).

O segundo fator contém apresenta os indicadores AP, AC e VPR, representando a Dimensão Econômica da Soja. Essa dimensão possui a segunda maior influência no ISPS, seguindo a ordem de explicação dos fatores. Tem-se que todos os valores para eles foram positivos, o que indica que assim que aumenta as áreas plantadas e colhidas, ocasiona em um aumento do valor da produção.

O terceiro fator é o que explica a menor variabilidade total dos dados, contendo os indicadores PRAD e Desmatamento, representando a Dimensão Ambiental. Apesar de ser a última em termos de importância para o ISPS, não significa que sua contribuição seja pequena. Como o valor da carga fatorial para o PRAD foi negativo e o Desmatamento positivo, temos que quanto mais se atua para recuperar as áreas degradadas, isso implica em uma redução da área desmatada, maior será a sustentabilidade, porém também significa que a região sofreu com o desmatamento.

Os escores dos fatores para cada observação foram obtidos pelo método de Bartlett, posteriormente padronizados para o intervalo 0 e 1, segundo a equação (2). Para obter o ISPS, utilizou-se a equação (1) com a variância total rotacionada.

A Tabela 5 mostra os resultados da regressão com coeficientes padronizados, com o objetivo de encontrar quais dos indicadores mais afetam a variabilidade do mais do ISPS. 
Tabela 5. Resultados da regressão múltipla com coeficientes padronizados entre o ISPS e seus indicadores.

\begin{tabular}{clll}
\hline Impacto no ISPS & Indicadores & Dimensão & Coeficientes \\
\hline $\mathbf{1}^{\mathbf{0}}$ & AC & Econômica & 0,26 \\
$\mathbf{2}^{\mathbf{0}}$ & PRAD & Ambiental & $-0,20$ \\
$\mathbf{3}^{\mathbf{0}}$ & VP & Social & $-0,18$ \\
$\mathbf{4}^{\mathbf{0}}$ & RPC & Social & 0,15 \\
$\mathbf{5}^{\mathbf{0}}$ & IDHM & Social & 0,14 \\
$\mathbf{6}^{\mathbf{0}}$ & IDHM E & Social & 0,14 \\
$\mathbf{7}^{\mathbf{0}}$ & EP & Social & $-0,14$ \\
$\mathbf{8}^{\mathbf{0}}$ & QP & Econômica & 0,13 \\
$\mathbf{9}^{\mathbf{0}}$ & VPR & Econômica & 0,12 \\
$\mathbf{1 0}^{\mathbf{0}}$ & IDHM R & Social & 0,09 \\
$\mathbf{1 1}^{\mathbf{0}}$ & P & Social & $-0,06$ \\
\hline
\end{tabular}

Fonte: Elaborado pelos autores.

Nesse caso, ao se analisar os impactos individuais dos indicadores, temos que a AC é a que mais influência na variabilidade do ISPS, seguido do PRAD e VP. Diante desses resultados, pode-se notar que individualmente as dimensões afetam o ISPS em ordem diferente no encontrado pela AF, isso ocorre pelo fato da AF analisar os efeitos conjuntos de todos os indicadores de cada dimensão.

A AC se mostrou mais importante devido estar diretamente relacionada ao valor produção da soja e a quantidade produzida, corroborando com o trabalho de Macedo et al. (2016) que afirma que maiores produções de soja, resultam em melhores resultados de sustentabilidade para o município produtor.

O PRAD se apresenta como o segundo que mais influência, pois segundo Almeida (2016), este plano contém diagnósticos e estudos que contribuem para a avaliação da área degradada pelas atividades econômicas, definindo, portanto, as medidas adequadas para a recuperação da área, a qual tornou-se indispensável a partir da criação do Cadastro Ambiental Rual CAR. Enfatiza-se que a área recuperada está diretamente relacionada ao aumento da sustentabilidade do município.

Em terceiro lugar se encontra o VP, que de acordo com Orsi (2009) só será possível alcançar a sustentabilidade quando o desenvolvimento for pautado, inclusive, na equidade social, demonstrada por intermédio da redução da vulnerabilidade a pobreza. Além disso, Weinhold, Killick e Reis (2013) salientam que a produção de soja colabora por essa redução nos municípios sojicultores.

\subsection{Aplicação da análise de cluster}

Após a obtenção do ISPS, utilizou-se a Análise de Cluster para agrupar os municípios que possuem nível de similaridade para sustentabilidade. A análise construiu quatro clusters, sendo que nessa fase pode-se comparar esse resultado com a classificação realizada pela EMBRAPA, no qual os municípios que estão no primeiro cluster serão classificados em nível crítico de sustentabilidade, no segundo cluster com nível baixo, no terceiro com médio e por último com alto.

\section{3 $O$ índice de sustentabilidade da produção da soja}

A Tabela 6 mostra as estatísticas descritivas para o ISPS em cada classificação alcançada. Note que o número de municípios classificados tanto em Crítico quanto Alto foram os que obtiveram menor quantidade de municípios, sendo 16 em 
cada, enquanto que a 35 foram classificados em Médio, em seguida, 25 classificados com nível Baixo de sustentabilidade. Na grande parte dos resultados a Média geométrica não se diferenciou da média, apenas no nível Crítica existe uma pequena diferença, o que já era de se esperar devido à natureza simétrica na padronização do índice.

Tabela 6. Estatística descritiva do ISPS dos municípios de acordo com a classificação de sustentabilidade.

\begin{tabular}{lllll}
\hline & ISPS Crítico & ISPS Baixo & ISPS Médio & ISPS Alto \\
\hline Tamanho da amostra & 16 & 25 & 35 & 16 \\
Mediana & 0.17 & 0.38 & 0.57 & 0.86 \\
Média Geométrica & 0.16 & 0.38 & 0.57 & 0.86 \\
Erro Padrão & 0.02 & 0.01 & 0.01 & 0.02 \\
Coeficiente de Variação & $48.96 \%$ & $9.46 \%$ & $11.47 \%$ & $9.45 \%$ \\
\hline
\end{tabular}

Fonte: Elaborado pelos autores.

A Figura 1 ilustra a variabilidade dos dados por meio dos Box-plot do ISPS em cada classificação, pode-se notar que em nenhum Box-plot houve a presença de outliers, não havendo municípios dentro de cada classificação que se destaque dos demais.

Figura 1. Box-plot do ISPS dos municípios de acordo com a classificação de sustentabilidade.

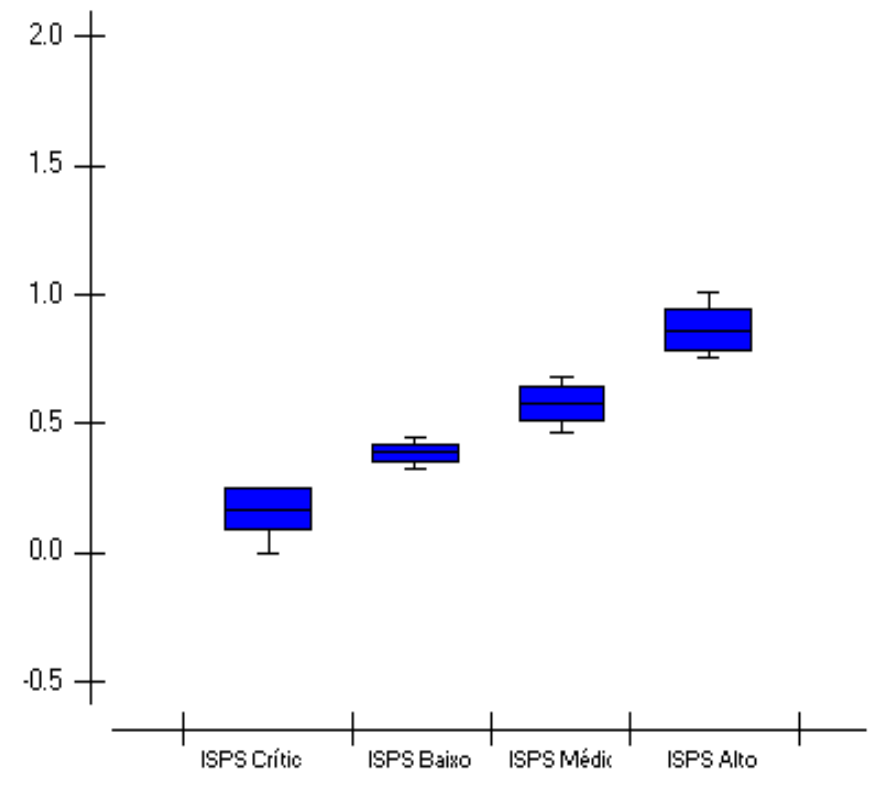

Fonte: Elaborado pelos autores.

É importante relacionar o nível de sustentabilidade com uma medida de desigualdade de renda, a Figura 2 associa a classificação do ISPS com o índice de Gini. Percebe-se que existe um pequeno decaimento do Gini de acordo com o aumento da sustentabilidade, isso corrobora com a afirmação de Weinhold, Killick e Reis (2013) de que a melhoria contínua das condições socioeconômicas é importante para reduzir as desigualdades na renda. Porém, no nível alto de sustentabilidade o município Campos de Júlio se apresenta como um outlier, isso pode ocorrer pelo fato de ser um dos que mais produzem soja no Estado, associado a uma população de 5.049 habitantes (IBGE, 2010) com a menor porcentagem nos indicadores de pobreza. Weinhold, Killick e Reis (2013) explicam que isso se justifica pela concentração da terra no setor rural, nesse caso específico existe uma alta desigualdade de renda entre as classes sociais mais altas, resultando no Gini de 0,62. 
Figura 2. Box-plot do Gini dos municípios de acordo com a classificação de sustentabilidade.

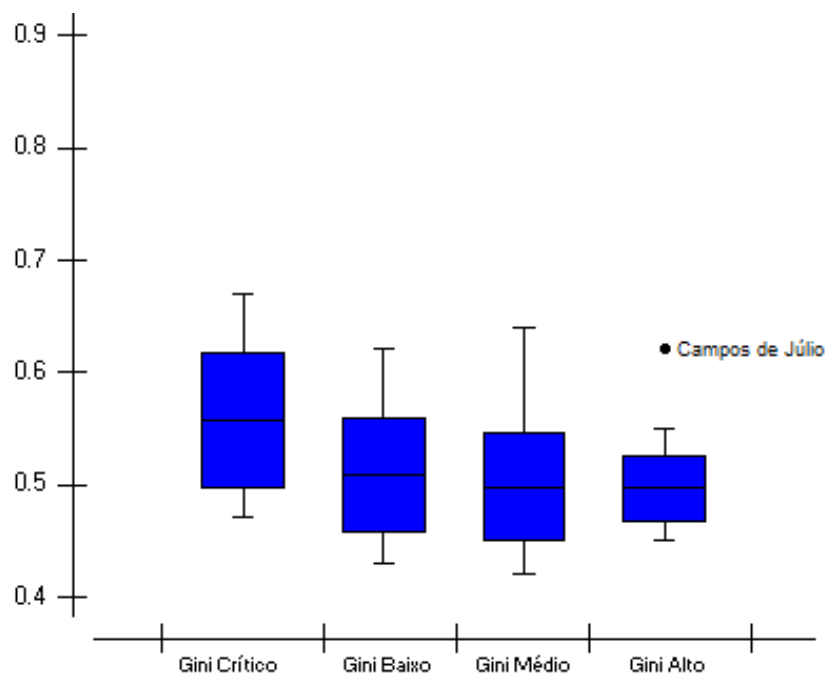

Fonte: Elaborado pelos autores.

Com o intuito de realizar a comparação entre o IDHM e o ISPS a Figura 3 mostra os resultados do Box-plot em que compara a variabilidade do IDHM dentre os níveis de sustentabilidade.

Figura 3. Box-plot do IDHM dos municípios de acordo com a classificação de sustentabilidade.

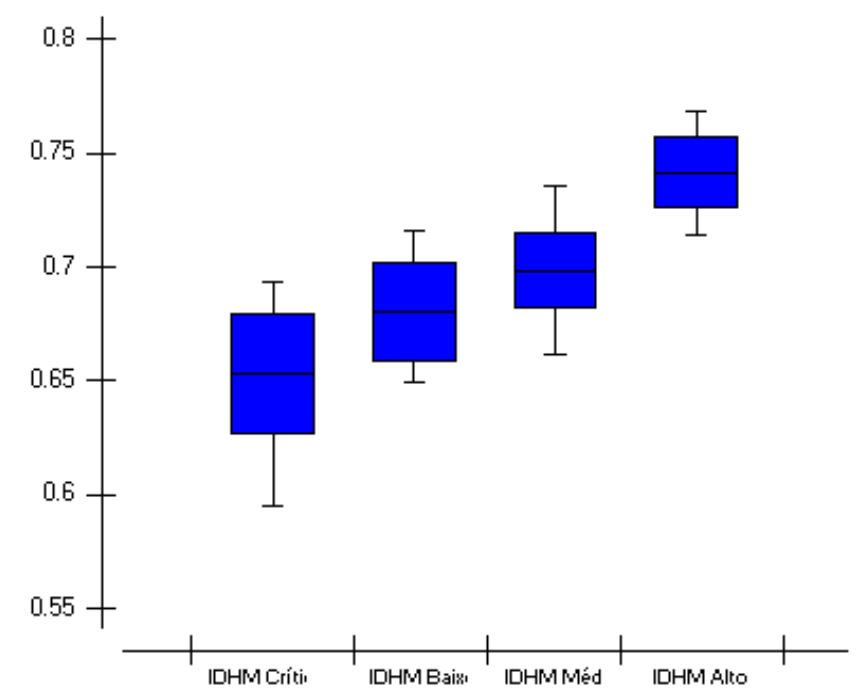

Fonte: Elaborado pelos autores.

É possível notar que não existem municípios outliers e que em termos de variabilidade o IDHM se manteve constantes entre os níveis. Observa-se que os valores do IDHM aumentam conforme os municípios foram classificados com níveis mais altos de sustentabilidade, associando as duas medidas de forma positiva.

Do ponto de vista da Quantidade Produzida, a Tabela 7 mostra sua estatística descritiva em seus níveis de sustentabilidade. A média se distancia muito da mediana, sugerindo uma distribuição assimétrica dos dados, porém ao se analisar a Figura 4 dos Box-plot, tem-se que existem alguns outliers presentes em cada classificação, por isso reflete nos altos valores dos coeficientes de variação. Sendo necessário a retirada desses municípios para uma análise mais correta. 
Tabela 7. Estatística descritiva da QP dos municípios de acordo com a classificação de sustentabilidade.

\begin{tabular}{lllll}
\hline & QP Crítico & QP Baixo & QP Médio & QP Alto \\
\hline Tamanho da amostra & 16 & 25 & 35 & 16 \\
Mediana & 7500 & 48000 & 121719 & 520511 \\
Média Aritmética & 26436.00 & 73552.96 & 176425.66 & 644028.06 \\
Erro Padrão & 14151.53 & 18735.17 & 33693.04 & 111727.81 \\
Coeficiente de Variação & $214.13 \%$ & $127.36 \%$ & $112.98 \%$ & $69.39 \%$ \\
\hline
\end{tabular}

Fonte: Elaborado pelos autores.

Na Figura 4 ao se relacionar a classificação de sustentabilidade com a Quantidade produzida, um município que chama atenção é o município Gaúcho do Norte, pois apesar de possuir maior Quantidade Produzida e Valor de Produção, com infraestrutura de apoio a produção de soja, como armazéns, foi classificado como crítico, possivelmente por possuir altos indicadores de pobreza. Os demais se comportaram como o esperado, estando classificados com maior sustentabilidade em consonância com sua produtividade de soja, o que corrobora com os resultados de Macedo et al. (2016).

Figura 4. Box-plot do QP dos municípios de acordo com a classificação de sustentabilidade.

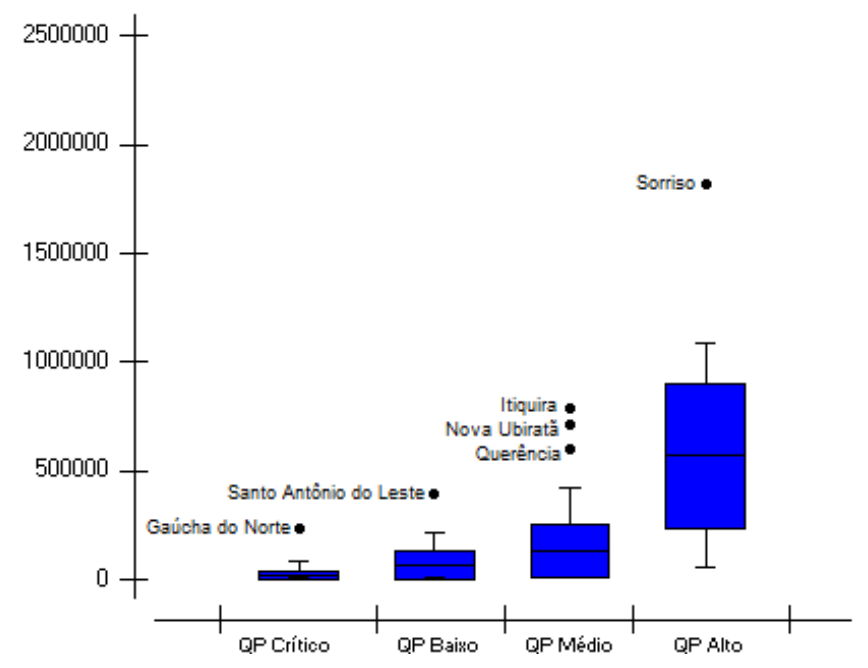

Fonte: Elaborado pelos autores.

A Tabela 8 mostra os resultados após a retirada dos outliers, pode-se notar que pouco se alterou as médias do ISPS, porém para QP agora a média se aproxima mais da mediana com uma diminuição no coeficiente de variação. Dessa forma, temos que nos níveis mais baixos de sustentabilidade produziram maior variabilidade. 
Tabela 8. Relação entre o ISPS e a Quantidade Produzida de Soja.

\begin{tabular}{c|ll|lll}
\hline Cluster & ISPS & & \multicolumn{4}{l}{ Quantidade Produzida } & \\
\hline & Média* $^{*}$ & Classificação & Média* & Mediana & Coeficiente de Variação* \\
\hline $\mathbf{1}$ & 0.168 & Crítico & 13083 & 7500 & $148.33 \%$ \\
$\mathbf{2}$ & 0.38208333 & Baixo & 60354.8333 & 48000 & $112.53 \%$ \\
$\mathbf{3}$ & 0.55030303 & Médio & 123611.515 & 121719 & $96.42 \%$ \\
$\mathbf{4}$ & 0.85133333 & Alto & 566003.267 & 520511 & $58.50 \%$ \\
\hline
\end{tabular}

Fonte: Elaborado pelos autores.

Os resultados indicam que, em média e mediana, os municípios com menor quantidade produzida de soja possuem os piores ISPS, sendo classificados em ordem crescente de sustentabilidade de acordo com sua produção. O coeficiente de variação revela que no nível crítico ocorreu a maior variabilidade registrada em relação a média, possuindo uma dispersão mais acentuada, pois mesmo todos estando em estado Crítico nem todos compartilham da mesma situação Econômica, Social e Ambiental. Entretanto, as demais classificações indicam que os municípios a qual pertencem podem ser mais semelhantes entre si enquanto as dimensões abordadas no estudo.

Segundo Macedo et al. (2016) isso acontece porque os efeitos do dinamismo econômico da sojicultura colaboram para a sustentabilidade tanto na dimensão econômica quanto na social e ambiental dos municípios mato-grossenses que detém as maiores produções de soja, assim, estes possuem os melhores resultados nas dimensões e maiores similaridades.

Enquanto que os municípios que detém baixa produção de soja, geralmente, apresentam como principal atividade produtiva a extração de madeira ou a pecuária, as quais são altamente nocivas para o meio ambiente, mas geram retornos econômicos dependendo da sua intensidade em cada município, ademais Santos (2016) afirma que as disparidades nas dimensões de sustentabilidade sugerem a vulnerabilidade de um desenvolvimento onde o setor agropecuário é o responsável pela oscilação econômica e o crescimento desigual entre os municípios.

\section{Considerações Finais}

Este estudo teve por objetivo analisar como a produção de soja influencia na sustentabilidade dos municípios do Estado do Mato Grosso, por meio da construção de um Índice de Sustentabilidade da Produção da Soja no ano de 2010.

Constatou-se que a dimensão social foi a que mais explicou a variabilidade total dos dados, deste modo seus indicadores, quando avaliados de forma conjunta, desempenharam uma maior influência no ISPS do que os demais. Assim, todos os indicadores de pobreza - vulnerável a pobreza, pobres e extremamente pobres -, são inversamente relacionados com a sustentabilidade, enquanto o IDHM possui uma relação direta, pode-se afirmar, então, que um IDHM elevado, associado com a redução da pobreza e da desigualdade contribuem significativamente para alcançar a sustentabilidade.

Nesse contexto, a dimensão econômica foi a segunda que mais influenciou no ISPS, averiguando que uma grande área plantada e colhida resulta no aumento da quantidade produzida e do valor de produção, que associados elevam a sustentabilidade econômica. A dimensão ambiental, por sua vez, ficou em terceira em termo de importância para o ISPS, apesar de sua imensa contribuição. Enfatiza-se com base nos indicadores selecionados - o PRAD e o Desmatamento-, que o aumento da recuperação das áreas degradadas e a redução do desmatamento, colaboram para a sustentabilidade. Em relação aos impactos individuais dos indicadores, constatou-se que a área colhida é a que mais influência na variabilidade do ISPS, seguido do PRAD e Vulnerável a pobreza.

Portanto, verificou-se, por intermédio do Índice de Sustentabilidade da Produção da Soja proposto, que a produção de soja contribui para a sustentabilidade dos municípios do Estado do Mato Grosso, uma vez que os municípios com maiores 
produções de soja como Sorriso, Lucas do Rio Verde, Campo Novo do Parecis apresentam um nível alto de sustentabilidade, assim como os municípios que não são grandes produtores, mas detém uma infraestrutura de apoio ao fomento da produção, como Rondonópolis.

\section{Referências}

ABIOVE - Associação Brasileira das Indústrias de Óleos Vegetais. Moratória da Soja - Safra 2015/2016. (2016). http://www.abiove.org.br/site/_FILES/Portugues/09112016-141009-relatorio_da_moratoria_da_soja_2015-16_gts.pdf.

Assad, E. D., Martins, S. C., \& Pinto, H. S. (2012). Sustentabilidade no Agronegócio Brasileiro. Coleção de Estudos sobre Diretrizes para uma Economia Verde no Brasil. Fundação Brasileira para o Desenvolvimento Sustentável - FBDS. http://fbds.org.br/fbds/IMG/pdf/doc553.pdf.

Barros, G. S. C. (2016). Agronegócio: como preservar e potencializar seu papel estratégico. CEPEA. http://www.cepea.esalq.usp.br/br/opiniaocepea/agronegocio-como-preservar-e-potencializar-seu-papel-estrategico.aspx.

CNA - Companhia Nacional de Abastecimento. (2017). Séries Históricas - Soja. http://www.conab.gov.br/conteudos.php?a=1252.

Costa, C. G. A., Cândido, G. A., \& Macedo, L. O. B. (2015). Análise descritiva e comparativa do Programa Soja Plus no Estado de Mato Grosso: uma abordagem a partir da responsabilidade social empresarial. Revista de Administração e Negócios da Amazônia, 7(2). https://doi.org/10.18361/21768366/rara.v8n3p292-314

Costa, A. A. V. M. R. (2010). Agricultura sustentável II: avaliação. Revista de Ciências Agrárias. Lisboa, 33(2), 75-89. http://www.scielo.mec.pt/pdf/rca/v33n2/v33n2a07.pdf.

Fávero, L. P., Belfiore, P., Silva, P., \& Chan, B. (2009). Análise de Dados: Modelagem Multivariada para Tomada de Decisões. Campos Elsevier, 1 ed.

Ferreira, D. F. (2011). Estatística Multivariada. UFLA, 2 ed.

Hair JR, J. F., Anderson, R. E., Tatham, R. L., \& Black, W. C. (2005). Análise Multivariada de Dados. Porto Alegre: Bookman, (5a ed.).

Hirakuri, M. H., Castro, C., Franchini, J. C., Debiasi, H., Procópio, S. O., \& Junior, A. A. B. (2014). Indicadores de sustentabilidade da cadeia produtiva da soja no Brasil. Embrapa Soja: $\quad$ Londrina. ttps://www.infoteca.cnptia.embrapa.br/bitstream/doc/990556/1/Indi cadoresdesustentabilidadedacadeiaprodutivadasojanoBrasil.pdf.

Hirakuri, M. H., Castro, C., Franchini, J. C., Debiasi, H., Procópio, S. O., \& Junior, A. A. B. (2015). Metodologia para avaliação de sustentabilidade da cadeia produtiva da soja no Brasil. Embrapa Soja: Londrina. https://ainfo.cnptia.embrapa.br/digital/bitstream/item/135948/1/doc365.pdf.

IBGE - Instituto Brasileiro de Geografia e Estatística. Indicadores de Desenvolvimento Sustentável. (2015). Estudos \& Pesquisas: informação geográfica. http://biblioteca.ibge.gov.br/visualizacao/livros/liv94254.pdf.

IBGE - Instituto Brasileiro de Geografia e Estatística. (2017). Censo 2010. https://censo2010.ibge.gov.br.

Jacobi, P. (1999). Meio ambiente e Sustentabilidade. In: O município no século XXI: cenários e perspectivas. CEPAM.175-183. http://www.franciscoqueiroz.com.br/portal/phocadownload/desenvolvimento\%20sustentavel.pdf.

Johnson, R. A., \& Wichern, D. W. (2007). Applied Multivariate Statistical Analysis. Upper Saddle River: Pearson Education, (6a ed.).

Kastens, J. H.; Brown, J. C., Coutinho, A. C., Bishop, C. R., \& Esquerdo, J. C. D. M. (2017). Soy moratorium impacts on soybean and deforestation dynamics in Mato Grosso, Brazil. Plos One, 12(4): E0176168. https://doi.org/10.1371/journal.pone.0176168.

Maccari, N. (2014). Environmental Sustainability and Human Development: A Greening of Human Development Index. https://papers.ssrn.com/sol3/papers.cfm?abstract_id=2426073.

Macedo, L. O. B, Costa, C. G. A., Silva, J. V. F, \& Cândido, G. A. (2016). Influências da produção de soja sobre a sustentabilidade dos Municípios do Estado de Mato Grosso - MT. Revista Espacios. Vol. 37 (Nº7). http://www.revistaespacios.com/a16v37n07/16370709.html.

Macedo, L. O. B, Cândido, G. A, Costa, C. G. A., \& Silva, J. V. F. (2016). Avaliação da Sustentabilidade dos municípios do Estado de Mato Grosso mediante o emprego do IDSM - índice de desenvolvimento sustentável para municípios. Revista Brasileira de Gestão e Desenvolvimento Regional. v. 12, n. 3, p. 323345, set-dez, Taubaté, SP. http://www.rbgdr.net/revista/index.php/rbgdr/article/view/2530/548.

Martins, M. F.; \& Cândido, G. A. (2008). Índice de Desenvolvimento Sustentável - IDS dos Estados brasileiros e dos municípios da Paraíba. João Pessoa: Edições SEBRAE.

MAPA - Ministério da Agricultura, Pecuária e Abastecimento. (2020). AGROSTAT - Estatísticas de Comércio Exterior do Agronegócio Brasileiro. http://indicadores.agricultura.gov.br/index.htm.

MAPA - Ministério da Agricultura, Pecuária e Abastecimento. (2020). Projeções do agronegócio: Brasil 2018/2019 a 2028/2029, projeções de longo prazo. Brasília, DF. http://www.agricultura.gov.br/assuntos/politica-agricola/todas-publicacoes-de-politica-agricola/projecoes-do-agronegocio/projecoes-doagronegocio-2018-2019-2028-2029. 
Research, Society and Development, v. 10, n. 13, e280101321406, 2021

(CC BY 4.0) | ISSN 2525-3409 | DOI: http://dx.doi.org/10.33448/rsd-v10i13.21406

Mueller, C. (1995). As contas Nacionais e os Custos Ambientais da Atividade Econômica. Revista Análise Econômica, 13(23), 66-99. http://seer.ufrgs.br/index.php/AnaliseEconomica/article/view/10523/6164.

Orsi, R. A. (2009). Reflexões sobre o desenvolvimento e a sustentabilidade: O que o IDH e o IDHM podem nos mostrar? Tese de Doutorado, Universidade Estadual Paulista, Rio Claro, São Paulo, Brasil. http://hdl.handle.net/11449/104400.

Quinhoneiro, F. H. F. (2015). Desenvolvimento de metodologia de análise de indicadores de sustentabilidade como ferramenta para tomada de decisão utilizando lógica fuzzy. Dissertação de Mestrado, Universidade de São Paulo, São Paulo, Brasil. https://doi.org/10.11606/D.85.2016.tde-17032016-090809.

Sachs, I. (1993). Estratégias de transição para o século XXI. In: Bursztyn, M. (Org.). Para Pensar o Desenvolvimento Sustentável. Brasiliense, p.29-56.

Santos, G. T. (2016). Índice de desenvolvimento sustentável dos municípios de Mato Grosso. Trabalho de Conclusão de Curso, Instituto Federal do Mato Grosso, Cuiabá, Brasil. http://tga.blv.ifmt.edu.br/media/filer_public/0d/45/0d45ea24-4aeb-4ef6-8aa9-50c3777199ca/tcc_vf_gisele_santos.pdf.

SEMA MT - secretaria de estado de meio ambiente do Mato Grosso. (2014). Metodologia para construção de indicadores. http://www.sema.mt.gov.br/index.php?option=com_content $\& v i e w=$ article \&id=2406\&Itemid=853 .

Sequinel, M. C. M. (2002). O modelo de sustentabilidade urbana de Curitiba: um estudo de caso. Dissertação de Mestrado, Universidade Federal de Santa Catarina, Florianópolis, Brasil. http://repositorio.ufsc.br/xmlui/handle/123456789/83553

Weinhold, D., Killick, E.. \& Reis, E. J. (2013). Soybeans, Poverty and Inequality in the Brazilian Amazon. World Development, 52, 132-143. http://dx.doi.org/10.1016/j.worlddev.2012.11.016. 